\title{
Mechanism for faster periodical
}

\section{Opinion}

Almost all researchers have been complaining that publishing takes too long. I do not have to provide any more references for this. New ideas, new findings cannot be heard, learned and referred to advance the science for at least a few months after they were produced. The period of research is long and slow. The efficiency is severely compromised. Scientific experiments are conducted on all new hardware. But the scientific publishing is still using the same old software with no new mechanism. Without knowing the most recent discovery, researchers are repeating the same experiments that had been finished a few months ago. Science is cycling in the same period for a few months without moving forward. I don't know how to calculate how much it costs science for the whole scientific community year after year.

But is there a mechanism for a faster period?

Each author has to swear that no part of the manuscript has been submitted to any other journal. This policy is actually for the protection of the journal. So the reviewers will not do the reviewing process in vain. The journal will publish something really novel. With this policy, the efficiency of the journal seems more important than the efficiency of science. The novelty of the journal seems more important than protection of the novelty of authors'.

The situation is worsened in the era of maximizing the impact factor. Authors want to try to submit from the highest impact factor journal downwards until the manuscript is eventually accepted. This is bad for science as well as scientists. Good science is delayed. Science is valued by the journal it is published on. And anything can happen during this lengthy waiting, reviewing, rejecting, resubmitting, revising and producing processes. The novelty can be scooped or even stolen.

A better way for both science and scientists is publishing before peer reviewing process. Or we can give scientists even more freedom by abolishing the reviewing process for the novelty that may not be easily understood currently. Authors Journal is probably the only journal that claims no reviewing process. ${ }^{1}$ Before all these new ideas are prevalent, here I want to propose a way to a faster period of science without touching the peer reviewing system. ${ }^{2}$ Scientists should be allowed to submit to a few journals simultaneously. If more than one journal accept the manuscript, authors can choose one of them to publish within certain time frame. If the authors want to publish as early as possible, they can also choose the first journal which accept the manuscript and withdraw from others. In this way, the waiting time is minimized for science and scientists. The impact factor can be maximized at the same time. So that the efficiency of scientific research will increase dramatically. The valuable research funding around the world from our beloved taxpayers will be used more efficiently.
Volume 4 Issue 4 - 2016

\author{
Youhe Gao \\ College of Life Science, Beijing Normal University, China
}

Correspondence: Youhe Gao, Department of Biochemistry and Molecular Biology, Beijing Normal University, Gene Engineering and Biotechnology Beijing Key Laboratory, Beijing, I00875, P. R. of China, Tel +86 1058804382

Email gaoyouhe@bnu.edu.cn

Received: November 24, 2016 | Published: November 28, 2016

What are the side effects? Some of reviewers' efforts may be wasted. This can also be solved if all the journals share the same group of reviewers but make different decisions based on the reviewers' recommendations. Anyway, loss will be still affordable one compare to the time wasted during the current publishing process. The overall result will be beneficial to science. To move a step further, we can simply allow more than one journal to publish the same paper. This is actually no harm to science. For a finding which has impacts on different areas, it should be allowed to reach readers from different fields by publishing on different journals. This only interferes the evaluation system of scientists by count how many paper a scientist publishes. This paper counting system is not used everywhere and should have been abolished long time ago. In a scientist's report card, he/she should only report the contribution rather than the number of papers published. We need new mechanism for faster periodicals and faster period of science.

\section{Acknowledgements}

This work was supported by the National Key Research and Development Program of China (2016YFC1306300); the National Basic Research Program of China (2013CB530850); and funds from Beijing Normal University (11100704, 10300-310421102).

\section{Conflict of interest}

The author is the chief architect of Authors Journal and chief editor of MOJPB.

\section{References}

1. Gao Y. Start the New Year with the Best Gift. Authors Journal. 2016;1(1):00001.

2. h t t p : / / b log. s c i e n c e n e t. c n / h o m e php? $\bmod =$ space $\&$ uid $=244733 \&$ do $=$ blog $\& \mathrm{id}=1011714$ 